Психология. Журнал Высшей школы экономики,

2017. T. 14. № 4. C. 679-697. DOI: 10.17323/1813-8918-2017-4-679-697

\title{
РОЛЬ ЛАТЕРАЛЬНОЙ АСИММЕТРИИ В ЗАДАЧЕ ПОДАВЛЕНИЯ ОТВЕТА ПО КОМПОНЕНТАМ ВЫЗВАННЫХ ПОТЕНЦИАЛОВ
}

\author{
Ю.А. МАРАКШИНА ${ }^{\text {a,b }}$, А.В. ВАРТАНОВ ${ }^{\mathrm{a}}$, Б.И. БЕСПАЛОВ
}

\begin{abstract}
${ }^{a}$ Московский государственный университет инени М.В. Ломоносова, 119991, Россия, Москва, Ленинские горы, д. 1

${ }^{5}$ Психологический институт Российской академии образовапия, 125009, Россия, Москва, ул. Моховая, ә. 9, стр. 4
\end{abstract}

\begin{abstract}
Резюме
Исследование посвящено проблеме реализации функции подавления когнитивного контроля у лиц с различными характеристиками латеральной асимметрии - доминантности левой или правой руки или глаза. Группы участников с ведущим правым и левым глазом, а также ведущей правой и левой рукой выполняли задачи Go/NoGo различной перцептивной сложности. В результате обнаружены значимые различия в амплитуде компонентов вызванных потенциалов N200 и Р300. Различия в амплитуде N200 в правом височном отведении обнаружены между участниками с правым и левым векторами латеральной асимметрии в двух задачах в обоих условиях (Go и NoGo) в отведении Т6 и могут свидетельствовать о независимости процессов цветовой обработки стимулов от необходимости подавления ответа, а также о том, что на раннем этапе обработки стимулов различия между группами касаются обработки их цвета. Различия в амплитуде компонента Р300 представлены в большем диапазоне отведений и могут быть связаны с перцептивной сложностью визуальных стимулов. Использование ресурсов когнитивного контроля различается у групп с право- и левосторонним векторами латеральной асимметрии. В задаче, где целевые стимулы находятся вне фона, больше различий регистрируется в условии, требующем подавления нерелевантного ответа (NoGo); в задаче, где стимулы интегрированы в фон, в условии, не требующем подавления (Go). Данное обстоятельство свидетельствует о различиях в осуществлении контроля у участников с различными типами латеральной асимметрии в связи с разными типами стимулов.
\end{abstract}

Ключевые слова: когнитивный контроль, вызванные потенциалы, Go/NoGo, доминантность руки, глазодоминантность.

Исследование когнитивного контроля как совокупности функций, позволяющих осуществлять целена- правленное поведение, является широко распространенным и перспективным направлением когнитивной

Исследование выполнено при поддержке Российского фонда фундаментальных исследований, проект № 13-06-00553а. 
психологии (Alvarez, Emory, 2006). В число данных функций входят подавление активности, нерелевантной текущей задаче, переключение между задачами, обновление релевантной информации (Miyake et al., 2000). Подавление нерелевантной активности изучается в ситуации выполнения испытуемыми различных задач (Kok, 1999). Одной из них является классическая задача Струпа: испытуемому предъявляется ряд слов, обозначающих цвета. При этом цвет шрифта, которым написаны эти слова, не совпадает с цветом, который обозначают слова. Перед испытуемым возникает две альтернативы ответа: прочитывать слово или называть цвет его букв. Тенденция прочитать слово является более привычной, поэтому способность назвать цвет букв характеризует величину подавления. Еще одним примером задачи на подавление нерелевантной активности является антисаккада. Задача испытуемого - реагировать на появление объектов в разных, заранее неизвестных сторонах визуального поля. Перед появлением объекта в противоположной стороне визуального поля возникает сигнал, который, с одной стороны, выступает подсказкой, что в следующий момент времени появится отслеживаемый объект, с другой стороны, задача испытуемого состоит в подавлении саккады в сторону этого стимула. Величина подавления определяется способностью испытуемого не отвечать саккадическими движениями глаз на стимулы-подсказки (так как в противном случае время, отводимое на отслеживание целевого объекта, будет упущено) и рассчитывается исходя из общего количества правильных ответов. Также широко распространенными при изучении подавления ответов можно назвать задачи в парадигме Go/NoGo: испытуемый должен реагировать на один стимул и тормозить ответ на другой, при нахождении в том же контексте. Примером может служить задача стоп-сигнал, stop-signal task (Logan, 1994). У испытуемого формируется установка на категоризацию объектов (например, разделять гласные и согласные буквы). В то же время дается инструкция не реагировать на объекты в случае появления стопсигнала, который может выступать в качестве звукового либо визуального (смена цвета буквы) предупреждения. Задержка стоп-сигнала по отношению к стимулу, определяемая исходя из индивидуального времени реакции, ведет к усложнению задачи. Величина подавления определяется исходя из ошибочных реакций на те стимулы, которые были помечены стоп-сигналом.

\section{Функциональная асимметрия и ее связь с подавлением ответов}

До сих пор остаются невыясненными многие особенности и механизмы когнитивного функционирования в зависимости от функциональной асимметрии органов, например, таких характеристик, как предпочтение ведущей руки и глазодоминантность. Левшество встречается в популяции с частотой $10.0-$ 12.6 и 5.9-10.4\% среди мужчин и женщин соответственно (Perelle, Ehrman, 1994). Известно, что в популяции леворуких чаще встречаются пациенты с неврологическими нарушениями - эпилепсией, 
трудностями в обучении (Bryden et al., 2005; Lewin et al., 1993). Однако существуют и данные, подтверждаюшие высокие способности левшей в выполнении когнитивных задач (Benbow, 1986; Halpern et al., 1998). Это касается в том числе задач, задействующих когнитивный контроль, и в частности подавление нерелевантных ответов. Так, лица с левой ведущей рукой показали меньшую степень интерференции в задаче Струпа и более успешно справлялись с ней по сравнению с правшами (Beratis et al., 2010). Стоит отметить, что эти данные противоречат более ранним исследованиям, показавшим бо́льшую успешность правшей в выполнении задачи Струпа, что может быть объяснено, однако, малым объемом выборки (Jorgenson et al., 1980). Также невозможно сделать окончательные выводы о мозговых механизмах подавления ввиду того, что в большей части исследований принимают участие добровольцы с правой ведущей рукой, однако существуют данные о том, что у них в этот процесс вовлечены в большей степени области правого полушария (Garavan et al., 1999). Существуют данные, подтверждающие бо́льшую успешность левшей в некоторых когнитивных задачах, в том числе включающих функцию подавления, что объясняется большей вовлеченностью в эти процессы правого полушария (Beratis et al., 2013). Наряду с этим представлены данные о преимушественном вовлечении левого полушария в успешное осуществление подавления ответов как у правшей, так и у левшей (Serrien, Sovijärvi-Spapé, 2013). Так или иначе, вопрос о мозговых механизмах подавления у лиц с левосторонней асимметрией остается открытым.

\section{Электрофизиологические корреляты подавления ответа в компонентах вызванных потенциалов}

Исследования с использованием задач в парадигме Go/NoGo связывают подавление ответа с двумя основными компонентами вызванного потенциала (Bokura et al., 2001). Первый из них - компонент N200, негативная волна в промежутке между 200 и 300 мс после стимула. Он отмечен только в ситуации предьявления стимулов, требующих подавления ответа (NoGo). Второй компонент - Р300, появляющийся в интервале от 300 до 600 мс. Он имеет бо́льшую латентность и амплитуду в условии NoGo, требуюшем подавления ответа, по сравнению с условием Go, где подавлять ответ не требуется.

Исследований различия между компонентами вызванных потенциалов у испытуемых с различными характеристиками латеральной асимметрии в ситуациях, где требуется подавление непроизвольного ответа, немного. В экспериментах с использованием антисаккад выявлено снижение амплитуды предсаккадической настройки в лобной области и повышение - в левой задневисочной области в группе участников с левым ведущим глазом по сравнению с участниками с правым ведущим глазом, кроме того, показана более значительная активация правого полушария у участников с левым ведущим глазом перед саккадами влево (Лазарев, Киренская, 2008). Полученные результаты интерпретируются 
как снижение вовлечения лобной коры наряду с повышением роли правого полушария и постцентральных областей коры у участников с левым ведущим глазом. В исследовании рабочей памяти, где сравнивалась активность в ситуациях инициации и подавления, наблюдалась бо́льшая амплитуда компонента Р50 фронтальных областей в ситуации инициации у участников с левой ведущей рукой (Beratis et al., 2009). Амплитуда этого компонента снижалась в ситуации подавления только у участников с левой ведущей рукой. У участников с правой ведущей рукой, напротив, в ситуации подавления активация увеличивалась.

Таким образом, эти данные косвенно подтверждают необходимость большей активации правого полушария при выполнении задач на подавление. Однако в связи с малой представленностью работ по исследованию подавления у испытуемых с характеристиками левосторонней асимметрии (в частности, на материале задач Go/ NoGo) существует необходимость выявления мозговых механизмов этого процесса. Данное исследование направлено на выделение компонентов вызванного потенциала, связанных с осуществлением функции подавления у испытуемых с левой ведущей рукой, а также у испытуемых с левым ведущим глазом на материале задачи в парадигме Go/NoGo.

\section{Характеристики выборки и методы исследования}

В исследовании приняли участие 27 участников мужского пола (средний возраст $-23.7 \pm 3.9$ года) с высшим (13 человек, 48\%), неокончен- ным высшим (11 человек, 41\%) и средним специальным образованием (3 человека, 11\%).

Все участники выполнили вспомогательные методики, позволяющие определить их ведущую руку и ведущий глаз. В качестве методики для определения ведущей руки использовался теппинг-тест в классическом варианте Ильина (Ильина, Ильин, 1975). Методика была представлена в графическом варианте и проводилась индивидуально с каждым участником. Процедура тестирования осуществлялась с помощью секундомера. В качестве измеряемого показателя теста рассчитывался коэффициент функциональной асимметрии по работоспособности левой и правой рук. Положительный знак коэффициента функциональной асимметрии свидетельствует о ведущей правой руке, отрицательный - о ведущей левой руке. После оценки результатов пройденных методик образовались группы участников с ведущей левой рукой ( 8 человек) и ведущей правой рукой (19 человек). В качестве методик для определения ведушего глаза использовались следующие пробы: «карта с дырой», «Подзорная труба», проба Розенбаха, «моргание» (Хомская и др., 1995). После оценки результатов пройденных методик были образованы группы участников с левым ведущим глазом (9 человек) и участников с правым ведущим глазом (18 человек). Группы по доминантности руки и глаза формировались независимо друг от друга и поэтому существенно пересекались (часто включали в себя тех же участников).

В основном эксперименте выполнялись две задачи на подавление 
ответа в парадигме Go/NoGo. Обе задачи отличались друг от друга по условиям восприятия. В первой задаче (Бабочка 1, Б1) участнику в центре черного экрана в течение 400 мс предъявлялось черно-белое изображение бабочки, служившее фоном. Затем появлялся стимул, требующий ответа или его подавления: цветные колечки справа или слева от бабочки. Смена цвета и местоположения колечка осушествлялась последовательно в квазислучайном порядке. Участнику давалась инструкция: «Нажимайте левую кнопку мыши, если зеленое колечко появляется слева от бабочки, и правую кнопку, если зеленое колечко появляется справа от бабочки. При этом во время появления красного колечка независимо от его местоположения ничего нажимать не нужно». Предъявляемый паттерн сохранялся на протяжении 1000 мс, в течение которых принимался ответ участника. Затем следовала пауза (800 мс), после которой предъявление осуществлялось в таком же порядке. Во второй задаче (Бабочка 2, Б2) стимул, требующий ответа или его подавления, был непосредственно интегрирован в фоновое изображение. Участнику, так же как и в первой задаче, в центре черного экрана в течение 400 мс предъявлялось чернобелое изображение бабочки, служившее фоном, затем появлялся стимул, требуюший ответа или его подавления: цветные пятнышки на левом или правом верхнем крыле. Смена цвета и местоположения пятна также осуществлялась в квазислучайном порядке. Участнику давалась инструкция: «Нажимайте левую кнопку мыши, если зеленое пятно появляет- ся на левом крыле, и правую кнопку, если зеленое пятно появляется на правом крыле. При этом во время появления красного пятна независимо от его местоположения ничего нажимать не нужно». Предъявляемый паттерн сохранялся на протяжении 1000 мс, в течение которых принимался ответ участника. После паузы (800 мс) предъявление осуществлялось в таком же порядке.

Таким образом, в обеих задачах необходимость нажимать на клавишу после появления зеленого колечка либо пятна выступала в качестве условия Go. При появлении красного колечка либо пятна у участника возникало непроизвольное желание нажать определенную клавишу для левого или правого пятна, но красный цвет являлся сигналом «стоп» (условие NoGo), на который должен был ориентироваться участник, чтобы произвольно «подавить» это желание. В каждой из задач было по 400 проб, предъявление осуществлялось с помощью программы Presentation (Neurobehavioral Systems, Inc.).

Измеряемые показатели: правильность нажатия и время реакции (BP) испытуемого на стимулы зеленого цвета (в миллисекундах). Во время выполнения задач основного эксперимента регистрировались вызванные потенциалы электроэнцефалограммы на момент предьявления цветного кольца/пятна. Для этого использовался энцефалограф «Нейро-КМ» фирмы «Статокин» (19 каналов, система 10-20\%, референтные электроды - мостоиды M1 и $\mathrm{M} 2$, заземляющий электрод на $\mathrm{Fpz}$, сопротивление $3-30$ кОм) с программой «Brain Win» для регистрации ЭЭГ и ВП. Для оценки значимо- 
сти различия в ВП при сравнении выделяемых групп испытуемых и задач использовался t-критерий Стьюдента для независимых выборок. Независимо анализировались центральные, теменные, височные и фронтальные отведения. Данные регионы представляют наибольший интерес, так как, по данным исследований, N200 регистрируется в нижней фронтальной, средне-цингулярной коре; Р300, кроме этих регионов, - еще в островковой, премоторной, фронтальной коре, в височных и фронтальных отделах (EnriquezGeppert et al., 2010).

\section{Результаты}

В результате сортировки отрезков ЭЭГ по номерам стимулов, удаления фрагментов, содержащих артефакты, и усреднения ВП для каждого из испытуемых было получено по четыpe ВП на предъявление стимула: для каждого условия (наличие/отсутствие подавления) и задачи Бабочка 1 (Б1) и Бабочка 2 (Б2). Далее эти индивидуальные ВП усреднялись (с оценкой значимости различия) по четырем группам участников: участники с правым ведущим глазом (ПГ), участники с левым ведущим глазом (ЛГ), участники с правой ведущей рукой (ПР), участники с левой ведущей рукой (ЛР). Для анализа ВП был взят отрезок записи, начиная с 200 мс до стимула (появление пятна/кольца) и заканчивая 500 мс после стимула. Отрезок до стимула не представляет собой ровную линию, так как во время него испытуемый реагировал на появление фонового изображения (бабочки). Мы анализировали, в первую очередь, компоненты N200 и P300.

На рисунке 1 представлены четыpe групповых ВП, по группам ЛР и ПР в условиях Go (отсутствие подавления ответа) и NoGo (подавление) в задаче Б1. В задаче Б1 амплитуда пика $\mathrm{N} 200$ в отведении Т6 достоверно $(p<0.05)$ различается между группами ПР и ЛР - выше у ПР участников.

Амплитуда пика N200 достоверно различается в отведении Т6 в обеих задачах, что показано в таблицах 1 и 2.

Компонент Р300 зарегистрирован в задачах Б1 и Б2 в различных отведениях. В задаче Б1 есть значимые различия в амплитуде Р300 между группами ПГ и ЛГ в условии Go (отсутствие подавления) по отведениям $\mathrm{Cz}, \mathrm{Fz}, \mathrm{C}$ 4, T3, T5, F3, F7. В этой

Таблица 1

Различия в амплитуде N200 в отведении Т6 в задаче Б1 $(p<0.05)$

\begin{tabular}{|c|c|c|c|c|}
\hline \multirow{2}{*}{ Групाы } & \multicolumn{4}{|c|}{ Условие } \\
\cline { 2 - 5 } & \multicolumn{2}{|c|}{ Go } & \multicolumn{2}{c|}{ NoGo } \\
\cline { 2 - 5 } & Латентность & t-значение & Латентность & t-значение \\
\hline ПР и ЛР & - & - & $313-317$ & от -2.149 до -2.086 \\
\hline ПГ и ЛГ & $214-229$ & от -2.301 до -2.033 & $\begin{array}{l}226-231 \\
291-310\end{array}$ & $\begin{array}{c}\text { от }-2.229 \text { до }-2.035 \\
\text { от }-2.736 \text { до }-2.014\end{array}$ \\
\hline
\end{tabular}


Рисунок 1

Вызванные потенциалы в отведении Т6 для задачи Б1, усредненные по группам участников с правой (сплошная линия и пунктир) и левой (штрихпунктир и мелкий пунктир) ведущей рукой на стимулы, требующие реакции (Go, нет подавления, сплошная линия и штрихпунктир для соответствующих групп участников) и не требующие реакции (NoGo, реакция подавления, пунктир и мелкий пунктир для соответствующих групп участников)

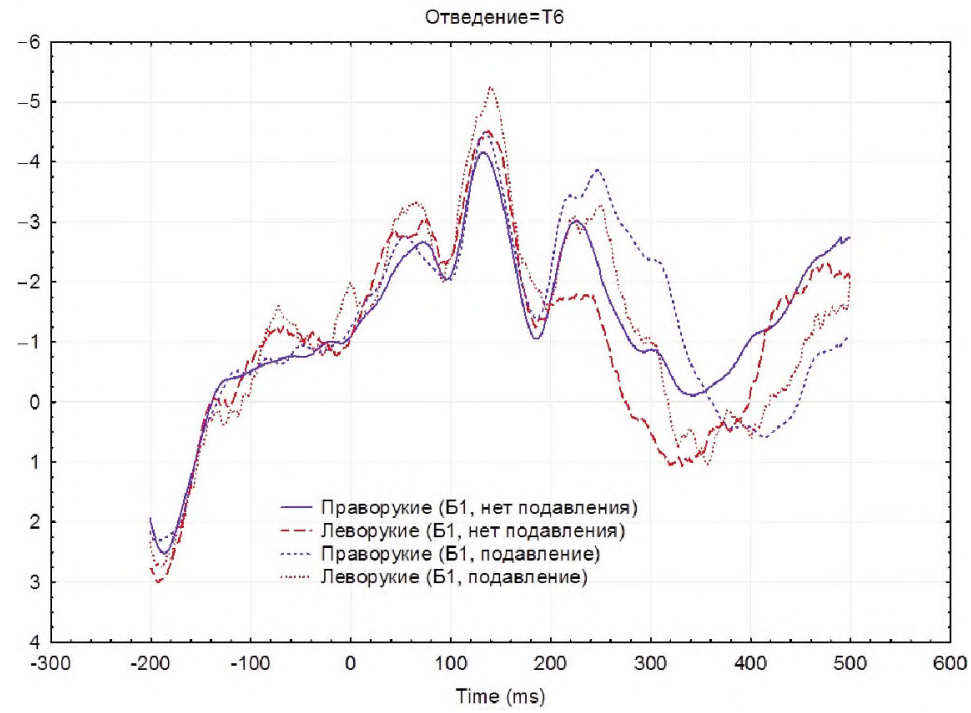

Примечание. Достоверные различия между групами ПР и ЛР участников обнаруживаются в амплитуде компонента N200 для стимулов, которые требуют подавления. При этом амплитуда N200 выше у ПР участников.

Таблица 2

Различия в амплитуде N200 в отведении Т6 в задаче $\mathbf{6} 2(p<0.05)$

\begin{tabular}{|c|c|c|c|c|}
\hline \multirow{2}{*}{ Группы } & \multicolumn{4}{|c|}{ Условие } \\
\cline { 2 - 5 } & \multicolumn{2}{|c|}{ Go } & \multicolumn{2}{c|}{ NoGo } \\
\cline { 2 - 5 } & Латентность & t-значение & Латентность & t-значение \\
\hline \multirow{2}{*}{ ПР и ЛР } & $209-225$ & от -2.282 до -2.039 & - & - \\
\hline ПГ и ЛГ & $244-254$ & от -2.112 до -2.013 & & от -2.855 до -2.070 \\
\hline
\end{tabular}

же задаче есть значимые различия в амплитуде P300 в условии NoGo (наличие подавления) в отведениях Pz, Cz, Fz, C3, T3, T4, T5, F3, F4, F7, F8 (см. таблицу 3).
На рисунке 2 для примера представлены четыре ВП по группам ЛГ и ПГ испытуемых в условиях отсутствия и наличия подавления в задаче Б1. Амплитуда пика Р300 в отведении 
Таблицаз

Различия в амплитуде Р300 между группами ПГ и ЛГ в задаче Б1 $(p<0.05)$

\begin{tabular}{|c|c|c|c|c|}
\hline \multirow{3}{*}{ Отведение } & \multicolumn{4}{|c|}{ Условие } \\
\hline & \multicolumn{2}{|r|}{ Go } & \multicolumn{2}{|r|}{ NoGo } \\
\hline & Латентность & t-значение & Латентность & t-значение \\
\hline $\mathrm{Pz}$ & - & - & $270-328$ & от -2.734 до -2.015 \\
\hline $\mathrm{Cz}$ & $492-499$ & от -2.187 до -2.036 & $255-276$ & от -2.522 до -2.009 \\
\hline \multirow{3}{*}{$\mathrm{Fz}$} & \multirow{3}{*}{$208-295$} & \multirow{3}{*}{ от -3.718 до -2.027} & $208-318$ & от -5.505 до -2.043 \\
\hline & & & $320-322$ & от -2.043 до -2.028 \\
\hline & & & $336-354$ & от -2.315 до -2.060 \\
\hline C3 & - & - & $253-294$ & от -3.313 до -2.039 \\
\hline $\mathrm{C} 4$ & $491-499$ & от -2.308 до -2.097 & - & - \\
\hline \multirow{6}{*}{ T3 } & \multirow{6}{*}{$478-485$} & \multirow{6}{*}{ от -2.240 до -2.036} & 209 & -2.093 \\
\hline & & & $223-229$ & от -2.164 до -2.033 \\
\hline & & & $261-265$ & от -2.090 до -2.022 \\
\hline & & & $268-300$ & от -3.272 до -2.054 \\
\hline & & & $323-334$ & от -2.232 до -2.020 \\
\hline & & & $357-370$ & от -2.194 до -2.008 \\
\hline \multirow{4}{*}{$\mathrm{T} 4$} & \multirow{4}{*}{-} & \multirow{4}{*}{-} & 221 & -2.048 \\
\hline & & & $267-295$ & от -3.190 до -2.056 \\
\hline & & & $322-341$ & от -3.045 до -2.080 \\
\hline & & & $420-432$ & от 2.046 до 2.288 \\
\hline \multirow{3}{*}{$\mathrm{T} 5$} & \multirow{3}{*}{352} & \multirow{3}{*}{2.018} & $365-370$ & от 2.061 до 2.148 \\
\hline & & & $397-402$ & от 2.016 до 2.234 \\
\hline & & & 404 & 2.028 \\
\hline F3 & $212-293$ & от -3.601 до -2.020 & $201-357$ & от -5.439 до -2.086 \\
\hline \multirow{5}{*}{ F4 } & \multirow{5}{*}{-} & \multirow{5}{*}{-} & 204 & -2.114 \\
\hline & & & 211 & -2.022 \\
\hline & & & $213-216$ & от -2.106 до -2.079 \\
\hline & & & $249-285$ & от -3.906 до -2.011 \\
\hline & & & $332-341$ & от -2.104 до -2.018 \\
\hline F7 & $265-277$ & от -2.105 до -2.007 & $267-288$ & от 2.046 до 2.288 \\
\hline \multirow{2}{*}{ F8 } & \multirow{2}{*}{-} & \multirow{2}{*}{-} & 204 & -2.021 \\
\hline & & & $246-272$ & от -2.768 до -2.049 \\
\hline
\end{tabular}


F4 достоверно различается между группами ПГ и ЛГ.

В задаче Б1 есть значимые различия в амплитуде Р300 между группами ПР и ЛР в условии Go (отсутствие подавления) по отведениям $\mathrm{Cz}$, Fz, C3, F3, F4, F8. В этой же задаче есть значимые различия в амплитуде Р300 между группами ПР и ЛР в условии NoGo (наличие подавления) в отведениях $\mathrm{Cz}, \mathrm{Pz}, \mathrm{Fz}, \mathrm{P} 3, \mathrm{C} 3, \mathrm{C} 4$, T3, T4, F3, F4, F7, F8 (см. таблицу 4).

В задаче Б2 есть значимые различия в амплитуде Р300 между группами ПГ и ЛГ в условии Go (отсутствие подавления) по отведениям Fz,
P3, C4, T3, T4, F3, F4, F8. В этой же задаче есть значимые различия в амплитуде P300 в условии NoGo (наличие подавления) в отведениях $\mathrm{Cz}, \mathrm{Fz}, \mathrm{C} 3, \mathrm{C} 4, \mathrm{~F} 3, \mathrm{~F} 4$ (см. таблицу 5).

В задаче Б2 есть значимые различия в амплитуде Р300 между группами ПР и ЛР в условии Gо (отсутствие подавления) по отведениям Pz, Cz, Fz, P3, P4, C3, C4, T3, T6, F3, F4. В этой же задаче есть значимые различия в амплитуде Р300 между группами ПР и ЛР в условии NoGo (наличие подавления) в отведениях Pz, Cz, P3, C3, C4, T3, Т4 (см. таблицу 6 ).

Рисунок 2

Вызванные потенциалы в отведении F4 для задачи Б1, усредненные по группам участников с правым (сплошная линия и пунктир) и левым (штрихпунктир и мелкий пунктир) ведущим глазом на стимулы, требующие реакции (Go, нет подавления, сплошная линия и штрихпунктир для соответствующих групп участников) и не требующие реакции (NoGo, реакция подавления, пунктир и мелкий пунктир для соответствующих групп участников)

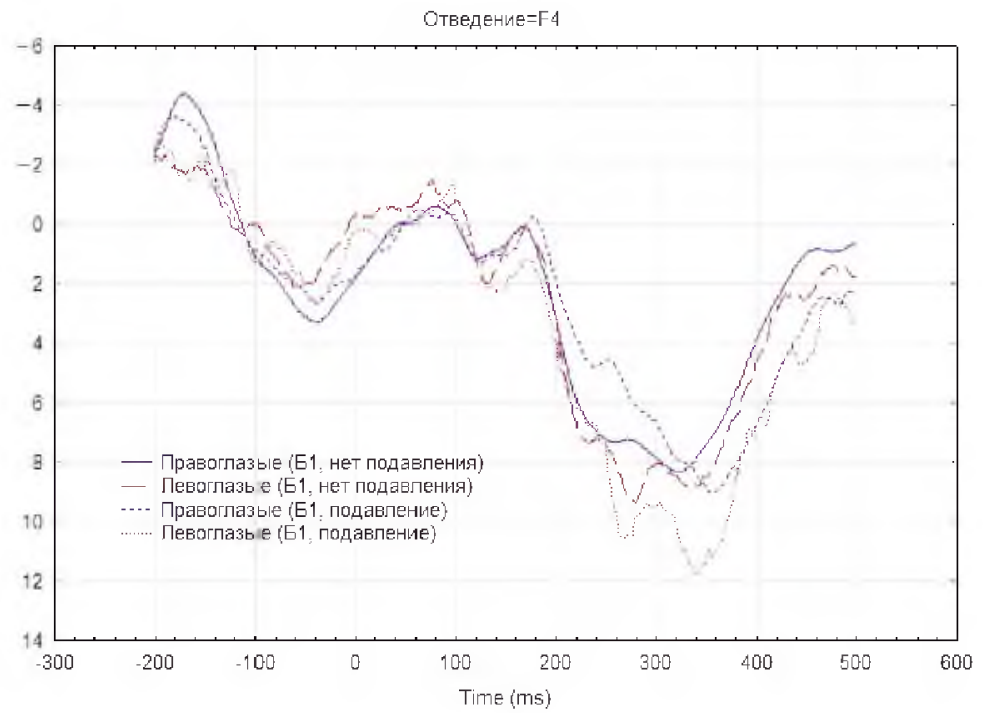

Примеиание. Достоверные различия между группами ПГ и ЛГ участников обнаруживаются в амплитуде компонента Р300 для стимулов, которые требуют подавления. При этом амплитуда Р300 больше у ЛГ участников. 
Таблица 4

Различия в амплитуде Р300 между группами ПР и ЛР в задаче Б1 $(p<0.05)$

\begin{tabular}{|c|c|c|c|c|}
\hline \multirow{3}{*}{ Отведение } & \multicolumn{4}{|c|}{ Условие } \\
\hline & \multicolumn{2}{|r|}{ Go } & \multicolumn{2}{|r|}{ NoGo } \\
\hline & Латентность & t-значение & Латентность & t-значение \\
\hline $\mathrm{Cz}$ & $263-290$ & от -2.696 до -2.027 & $259-295$ & от -3.137 до -2.007 \\
\hline $\mathrm{Pz}$ & - & - & $266-299$ & от -3.210 до -2.013 \\
\hline \multirow{2}{*}{$\mathrm{Fz}$} & \multirow{2}{*}{$254-300$} & \multirow{2}{*}{ от -3.736 до -2.031} & $258-316$ & от -4.759 до -2.075 \\
\hline & & & $384-391$ & от -2.090 до -2.019 \\
\hline P3 & - & - & $289-290$ & от -2.048 до -2.019 \\
\hline $\mathrm{C} 3$ & $254-292$ & от -2.293 до 2.010 & $264-290$ & от -3.306 до -2.027 \\
\hline \multirow{2}{*}{$\mathrm{C} 4$} & \multirow{2}{*}{ - } & \multirow{2}{*}{-} & $264-286$ & от -2.705 до -2.049 \\
\hline & & & $474-483$ & от 2.027 до 2.155 \\
\hline \multirow{5}{*}{$\mathrm{T} 3$} & \multirow{5}{*}{ - } & \multirow{5}{*}{ - } & $270-287$ & от -2.332 до-2. 007 \\
\hline & & & $368-400$ & от -3.522 до -2.010 \\
\hline & & & $412-435$ & от -2.962 до -2.040 \\
\hline & & & $460-467$ & от -2.297 до -2.033 \\
\hline & & & $494-496$ & от -2.134 до -2.034 \\
\hline \multirow{2}{*}{$\mathrm{T} 4$} & \multirow{2}{*}{ - } & \multirow{2}{*}{ - } & $328-330$ & от -2.056 до -2.020 \\
\hline & & & $335-337$ & От -2.050 до -2.015 \\
\hline \multirow{2}{*}{ F3 } & $253-300$ & от -3.620 до -2.042 & $258-300$ & от -4.417 до- 2.069 \\
\hline & - & - & $381-398$ & от -2.352 до -2.036 \\
\hline F4 & $260-296$ & от -3.490 до -2.010 & $258-301$ & от -3.833 до -2.027 \\
\hline F7 & - & - & $276-286$ & от -2.330 до -2.090 \\
\hline \multirow{2}{*}{ F8 } & \multirow{2}{*}{$273-285$} & \multirow{2}{*}{ от -2.188 до -2.007} & $264-278$ & от -2.259 до -2.042 \\
\hline & & & $465-490$ & от 2.064 до2.942 \\
\hline
\end{tabular}

На рисунке 3 для примера представлены четыре ВП по группам ЛР и ПР испытуемых в условиях отсутствия и наличия подавления в задаче Б2. Амплитуда пика Р300 в отведении F4 достоверно различается между группами ПР и ЛР.

Кроме анализа компонентов ВП, для каждого участника оценивалось среднее время реакции (ВР) в условии Go. Затем оценивалось среднее ВР для каждой из четырех групп (ЛГ, ПГ, ЛР, ПР). Различия ВР между группами оценивались по непараметрическому критерию Манна-Уитни. Данные представлены в таблице 7. При сравнении групп ПР и ЛР в задачах Б1 и Б2 различия оказались 
Различия в амплитуде Р300 между группами ПГГ и ЛГ в задаче Б2 $(p<0.05)$

\begin{tabular}{|c|c|c|c|c|}
\hline \multirow{3}{*}{ Отведение } & \multicolumn{4}{|c|}{ Условие } \\
\hline & \multicolumn{2}{|r|}{ Go } & \multicolumn{2}{|r|}{ NoGo } \\
\hline & Латентность & t-значение & Латентность & t-значение \\
\hline $\mathrm{Cz}$ & - & - & $466-497$ & от -2.447 до -2.034 \\
\hline \multirow{2}{*}{$\mathrm{Fz}$} & \multirow{2}{*}{$471-485$} & \multirow{2}{*}{ от -2.371 до -2.018} & $262-277$ & от -2.287 до -2.013 \\
\hline & & & $443-493$ & от -2.931 до -2.017 \\
\hline P3 & $299-311$ & от 2.007 до 2.102 & - & - \\
\hline \multirow{3}{*}{$\mathrm{C} 3$} & \multirow{3}{*}{-} & \multirow{3}{*}{-} & $454-456$ & от -2.120 до -2.040 \\
\hline & & & 458 & -2.012 \\
\hline & & & $497-498$ & от -2.028 до -2.040 \\
\hline \multirow{2}{*}{$\mathrm{C} 4$} & $470-473$ & от -2.094 до -2.022 & \multirow{2}{*}{$471-499$} & \multirow{2}{*}{ от -2.505 до -2.033} \\
\hline & 475 & -2.043 & & \\
\hline T3 & $245-259$ & от -2.958 до -2.073 & - & - \\
\hline $\mathrm{T} 4$ & $306-372$ & от 2.008 до 3.834 & - & - \\
\hline \multirow{2}{*}{ F3 } & $244-264$ & от -2.651 до -2.020 & \multirow{2}{*}{$449-469$} & \multirow{2}{*}{ от -2.644 до -2.027} \\
\hline & $470-493$ & от -2.427 до -2.027 & & \\
\hline \multirow{2}{*}{ F4 } & $455-488$ & от -2.762 до -2.038 & 457 & -2.008 \\
\hline & $490-491$ & от -2.047 до -2.038 & $459-487$ & от -2.670 до -2.043 \\
\hline F8 & $329-345$ & от 2.007 до 2.247 & - & - \\
\hline
\end{tabular}

минимальны и незначимы ( $U=73$, $p>0.05)$. При сравнении групп ПГ и ЛГ в задаче Б1 ВР у ЛГбыло на 30 мс больше, чем у ПГ, однако различия были также незначимы $(U=59, p>0.05)$. В задаче Б2 ВР у ЛГ было на 33 мс больше, значимость различий была на уровне тенденции $(U=47, p=0.08)$.

\section{Обсуждение}

Нам удалось обнаружить наличие компонента N200 в обеих задачах. N200 характеризует процессы когнитивного контроля, связанного с подавлением (Heil et al., 2000). В процессе выполнения задач пик
N200 появляется как в условии Go, так и в условии NoGo в правой височной области (отведение Т6). Появление пика может быть связано с опознанием стимулов, включающим в себя дифференцировку его цвета (зеленое или красное пятно) (Barnett, 2008). Дифференцировка цвета стимула происходит в правом полушарии, что требует меньших когнитивных затрат от лиц с левосторонним вектором асимметрии. Лицам с правосторонним вектором асимметрии, напротив, может потребоваться больше ресурсов для этой операции. Проведенные ранее исследования показали, что N200 
Различия в амплитуде Р300 между группами IIP и ЛР в задаче $62(p<0.05)$

\begin{tabular}{|c|c|c|c|c|}
\hline \multirow{3}{*}{ Отведение } & \multicolumn{4}{|c|}{ Условие } \\
\hline & \multicolumn{2}{|r|}{ Go } & \multicolumn{2}{|r|}{ NoGo } \\
\hline & Латентность & t-значение & Латентность & t-значение \\
\hline \multirow{2}{*}{$\mathrm{Pz}$} & $272-368$ & от -3.072 до -2.010 & \multirow{2}{*}{$272-330$} & \multirow{2}{*}{ от -3.177 до -2.094} \\
\hline & $389-405$ & от -2.248 до -2.012 & & \\
\hline \multirow{3}{*}{$\mathrm{Cz}$} & $202-223$ & от -2.482 до -2.052 & $272-341$ & от -3.413 до -2.036 \\
\hline & $253-365$ & от -3.403 до -2.007 & \multirow{2}{*}{$364-387$} & \multirow{2}{*}{ от -2.203 до -2.015} \\
\hline & $385-407$ & от -2.206 до -2.013 & & \\
\hline $\mathrm{Fz}$ & $282-329$ & от -2.798 до -2.020 & - & - \\
\hline \multirow{2}{*}{ P3 } & 298 & -2.007 & \multirow{2}{*}{$291-326$} & \multirow{2}{*}{ от -2.698 до -2.028} \\
\hline & 300 & -2.011 & & \\
\hline \multirow{5}{*}{$\mathrm{P} 4$} & $282-335$ & от -2.935 до -2.007 & \multirow{5}{*}{-} & \multirow{5}{*}{-} \\
\hline & $349-350$ & от -2.025 до -2.007 & & \\
\hline & $352-360$ & от -2.039 до -2.015 & & \\
\hline & $362-363$ & от -2.011 до -2.008 & & \\
\hline & $388-394$ & от -2.205 до -2.025 & & \\
\hline \multirow{2}{*}{ C3 } & $207-375$ & от -3.474 до -2.009 & \multirow{2}{*}{$262-330$} & \multirow{2}{*}{ от -3.016 до -2.043} \\
\hline & $382-412$ & от -2.608 до -2.009 & & \\
\hline \multirow{3}{*}{$\mathrm{C} 4$} & $206-222$ & от -2.351 до -2.027 & \multirow{3}{*}{$282-339$} & \multirow{3}{*}{ от -2.800 до -2.009} \\
\hline & $269-332$ & от -2.869 до -2.010 & & \\
\hline & $394-409$ & от -2.171 до -2.008 & & \\
\hline \multirow{5}{*}{$\mathrm{T} 3$} & $216-233$ & от -2.520 до -2.016 & \multirow{5}{*}{$290-326$} & \multirow{5}{*}{ от -2.800 до -2.019} \\
\hline & $253-354$ & от -2.933 до -2.040 & & \\
\hline & 357 & -2.015 & & \\
\hline & $360-374$ & от -2.169 до -2.012 & & \\
\hline & $390-414$ & от -2.799 до -2.011 & & \\
\hline $\mathrm{T} 4$ & - & - & 482 & 2.022 \\
\hline T6 & $310-336$ & от -2.558 до -2.010 & - & - \\
\hline F3 & $276-335$ & от -3.453 до -2.045 & - & - \\
\hline F4 & $280-332$ & от -2.664 до -2.012 & - & - \\
\hline
\end{tabular}


Рисунок 3

Вызванные потенциалы в отведении F4 для задачи 62, усредненные по группам участников с правой (сплошная линия и пунктир) и левой (штрихпунктир и мелкий пунктир) ведущей рукой на стимулы, требующие реакции (Go, нет подавления, сплошная линия и штрихпунктир для соответствующих групп участников) и не требующие реакции (NoGo, реакция подавления, пунктир и мелкий пунктир для соответствующих групп участников)

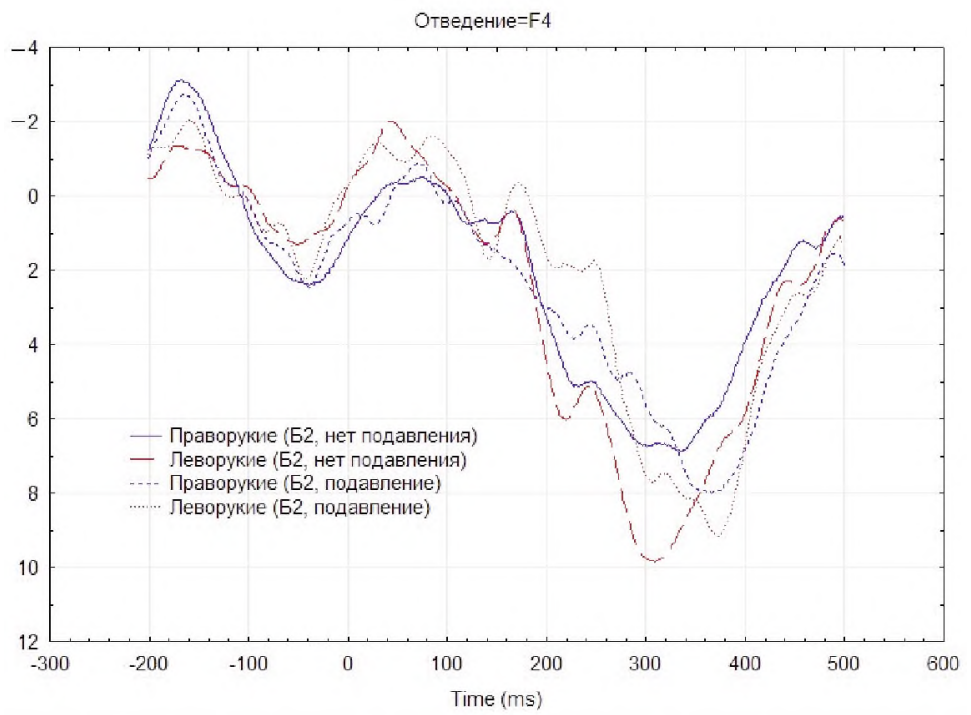

Примечание. Достоверные различия между группами ПР и ЛР участников обнаруживаются в амплитуде компонента Р300 для стимулов, которые не требуют подавления. При этом амплитуда Р300 больше у ЛР участников.

Таблица 7

Среднее время реакции в группах ЛГ и ІІГ, ЛР и ІІР в задачах Б1 и Б2

\begin{tabular}{|c|c|c|c|c|}
\hline Группы & $\begin{array}{c}\text { Среднее ВР в } \\
\text { задаче Б1 } \\
\text { (мс) }\end{array}$ & $\begin{array}{c}\text { Среднее ВР } \\
\text { в задаче Б2 } \\
\text { (мс) }\end{array}$ & $\begin{array}{c}\text { Различия среднего ВР } \\
\text { между группами в } \\
\text { задаче Б1 (мс) }\end{array}$ & $\begin{array}{c}\text { Различия среднего ВР } \\
\text { между группами в } \\
\text { задаче Б2 (мс) }\end{array}$ \\
\hline ЛГ & 410 & 430 & \multirow{2}{*}{30} & \multirow{2}{*}{33} \\
\hline$\Pi \Gamma$ & 380 & 397 & & \\
\hline ЛР & 393 & 403 & \multirow{2}{*}{5} & \multirow{2}{*}{7} \\
\hline ПР & 388 & 410 & & \\
\hline
\end{tabular}

появляется в условии NoGo только при выполнении визуальной задачи и не регистрируется при выполнении аудиальной (Falkenstein et al., 1995). Можно говорить о независимости возникновения N200 от необходимости подавлять или не подавлять ответ. Различия между группами участников с различными векторами латеральной асимметрии указывают 
на то, что процессы цветовой обработки осуществляются у лиц с левосторонними характеристиками с меньшими затратами ресурсов вне зависимости от наличия дополнительной нагрузки в виде осуществления подавления нерелевантных ответов. Появление N200 в обоих условиях (Go и NoGo) может свидетельствовать о важности этого компонента как для подавления, так и для инициации ответа, что согласуется с ранними исследованиями, предположившими, что N200 связан с мониторингом конфликта (Donkers, van Boxtel, 2004). Подтверждается значимость N200 для инициации действия (Bruin, Wijers, 2002).

Пик Р300 связан с процессом принятия решения и регистрируется обычно в теменной коре при активации непроизвольного внимания и во фронтальной коре при вовлечении произвольного, целенаправленного внимания (Li et al., 2010). Эти данные согласуются с нашими результатами: Р300 зарегистрирован как в париетальных, так и во фронтальных отведениях. Нам удалось обнаружить, что в обеих задачах различия в амплитуде Р300 между группами участников с левым и правым векторами латеральной асимметрии выражены по многим отведениям, широко распределенным по мозгу: теменным, височным, центральным, фронтальным. Однако в задаче Б1 различия выражены по большему количеству отведений в условии NoGo. B то же время в задаче Б2 различия более выражены в условии Go. Можно предположить, что задачи различаются перцептивной сложностью. В задаче Б1 стимулы, требующие ответа или подавления нежелательной реакции, находятся вне фонового изображения бабочки (колечки, появляющиеся сбоку от крыльев), в то время как в задаче Б2 стимулы интегрированы в фон (пятнышки на крыльях). В связи с тем, что целевые стимулы в задаче Б2 интегрированы в фон, это может потребовать больше ресурсов по их дифференциации для успешной инициализации ответа, что требует бо́льших затрат когнитивных ресурсов в условии Gо. В задаче Б1 отсутствует интеграция стимулов с фоном, их легче перцептивно различать, поэтому ресурсы затрачиваются на условие NoGo, требующее торможения ответа. Косвенное подтверждение предположению о связи перцептивной сложности визуальной задачи и различий в Р300 можно найти в исследованиях с использованием fMRI-данных, где обнаружены различия в паттернах мозговой активации при выполнении задач Go/NoGo различной степени сложности (Simmonds et al., 2008).

Наличие различий в амплитуде Р300 отчасти согласуется с данными по ВР: среднее ВР в условии, не требуюшем подавления ответа, у ЛГ на 33 мс больше, чем у ПГ, в задаче Б2 (хотя различия незначимы, выражена только тенденция). Таким образом, электрофизиологические показатели позволяют более точно дифференцировать различия в осуществлении когнитивного контроля у лиц с различными вариантами латеральной асимметрии.

\section{Выводы}

Различия в амплитуде N200 в группах участников с право- и левосторонним векторами асимметрии в 
правой височной области (отведение T6) в обоих условиях (Go и NoGo) могут свидетельствовать о разных процессах цветовой обработки стимулов в данных группах, а также о том, что они не зависят от необходимости подавлять ответ.

Различия в амплитуде Р300 в группах участников с право- и левосторонним векторами асимметрии представлены по многим отведениям (фронтальным, теменным, височным и центральным) в обоих условиях (Gо и NoGo).
Активация ресурсов когнитивного контроля различается у групп с правои левосторонним векторами латеральной асимметрии. Вклад фронтальных и других областей мозга в функцию подавления нерелевантного ответа связан с перцептивной сложностью задачи. В задаче, где целевые стимулы находятся вне фона, больше различий регистрируется в условии, требующем подавления нерелевантного ответа (NoGo). B задаче, где стимулы интегрированы в фон, - в условии, не требующем подавления (Go).

\section{Литература}

Ильина, М. Н., Ильин, Е. П. (1975). Об одном из условий диагностирования силы нервной системы по возбуждению с помощью теппинг-теста. В кн. Психофизиологические особенности спортивной деятельности: Сборник науиных работ (с. 183-186). Л.: ЛГПИ.

Лазарев, И. Е., Киренская, А. В. (2008). Влияние фактора ведущего глаза на характеристики саккад и медленных потенциалов ЭЭГ. Физиология человека, 34(2), 23-33.

Хомская, Е. Д., Привалова, Н. Н., Ениколопова, Е. В., Ефимова, И. В., Степанова, О. Б., Горина, И. С. (1995). Методы оценки межполуиарной асимметрии и межполуиарного взаимодейстеия. М.: Изд-во Московското университета.

Ссылки на зарубежные источники см. в разделе References после англоязыиного блока.

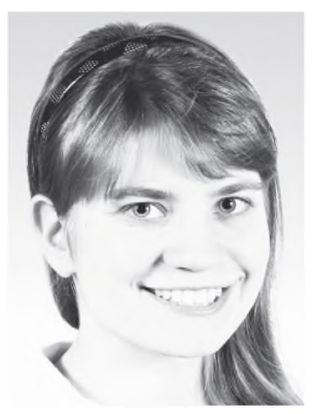

Маракшина Юлия Александровна - аспирант, факультет психологии, Московский государственный университет имени М.В. Ломоносова; научный сотрудник, Психологический институт Российской академии образования.

Сфера научных интересов: психофизиология, механизмы когнитивного контроля, внимания и рабочей памяти.

Контакты: retalika@yandex.ru 

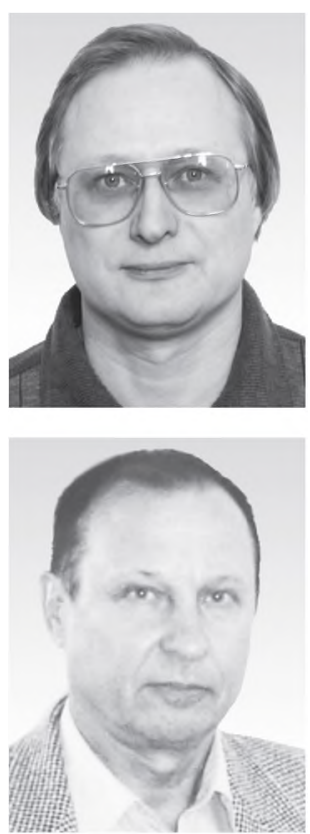

Вартанов Александр Валентинович - старший научный сотрудник, факультет психологии, Московский государственный университет имени М.В. Ломоносова, кандидат психологических наук.

Сфера научных интересов: психофизиология, механизмы семантики, сознания и высших психических функций.

Контакты: a v vartanov@mail.ru

Беспалов Борис Иванович - старший научный сотрудник, факультет психологии, Московский государственный университет имени М.В. Ломоносова, кандидат психологических наук.

Сфера научных интересов: культурно-деятельностная психология, психофизиологическая проблема, логико-математические модели познавательных действий.

Контакты: bespalovb@mail.ru

\title{
Role of the Lateral Asymmetry in Response Inhibition Task in Event-Related Potentials Components
}

\author{
J.A. Marakshina ${ }^{\mathrm{a} b \mathrm{~b}}$, A.V. Vartanov ${ }^{\mathrm{a}}$, B.I. Bespalov ${ }^{\mathrm{a}}$ \\ ${ }^{a}$ Lomonosov Moscow State University, 1 Leninskie Gory, Moscow, 119991, Russian Federation \\ ${ }^{b}$ Psychological Institute of Russian Academy of Education, 9/4 Mohovaya str., Moscow, 125009, Russian \\ Federation
}

\begin{abstract}
The research is dedicated to the issue of realization of inhibition as the function of cognitive control in people with various characteristics of lateral asymmetry - right or left hand or eye dominance. Groups of the subjects with right or left dominant eye, as well as right- or lefthanded solved the problems of Go/NoGo of various perceptual complexity. As a result, significant differences were found in the amplitude of components of evoked potentials N200 and P300. The differences in the amplitude N200 in the right temporal lead were found between the subjects with the right and left vectors of lateral asymmetry in 2 tasks in both conditions (Go and NoGo) in the lead T6, and they may speak for the independence of color processing of stimuli from the need to inhibit the response, as well as for the fact that in the early stage of processing the stimuli there are differences in color processing. The differences in the amplitude of the component P300 are seen in a wide range of leads and may be linked to perceptual complexity of the visual stimuli. The usage of cognitive control responses varies in groups with right and left vectors of lateral asymmetry. In the task where the relevant stimuli are out of the context, more differences are reg-
\end{abstract}


istered in the condition that demands inhibition of the irrelevant response (NoGo); in the task where stimuli are integrated in the context, more differences are registered in the condition that doesn't demand the inhibition (Go). This fact speaks for the differences in performance of control in subjects with various types of lateral asymmetry, when they answer various types of stimuli.

Keywords: cognitive control, event-related potentials, Go/NoGo, handedness, eye dominance.

\section{References}

Alvarez, J. A., \& Emory, E. (2006). Executive function and the frontal lobes: A meta-analytic review. Neuropsychology Review, 16(1), 17-42. doi:10.1007/s11065-006-9002-x.

Barnett, K. J. (2008). Colour knowledge: The role of the right hemisphere in colour processing and object colour knowledge. Laterality, 13(5), 456-467. doi:10.1080/13576500802146387

Benbow, C. P. (1986). Physiological correlates of extreme intellectual precocity. Neuropsychologia, 24(5), 719-725. doi:10.1016/0028-3932(86)90011-4

Beratis, I. N., Rabavilas, A. D., Kyprianou, M., Papadimitriou, G. N., \& Papageorgiou, C. (2013). Investigation of the link between higher order cognitive functions and handedness. Journal of Clinical and Experimental Neuropsychology, 35(4), 393-403. doi:10.1080/13803395.2013.778231

Beratis, I. N., Rabavilas, A., Nanou, E. D., Hountala, C., Maganioti, A. E., Capsalis, C. N., \& Papageorgiou, C. (2009). Effect of initiation-inhibition and handedness on the patterns of the P50 event-related potential component: A low resolution electromagnetic tomography study. Behavioral and Brain Functions, 5(51). doi:10.1186/1744-9081-5-51. Retrieved from http://behavioralandbrainfunctions.biomedcentral.com/articles/10.1186/1744-9081-5-51

Beratis, I. N., Rabavilas, A., Papadimitriou, G. N., \& Papageorgiou, C. (2010). Effect of handedness on Stroop Colour Word Task. Laterality, 15(6), 597-609. doi:10.1080/13576500903071104

Bokura, H., Yamaguchi, S., \& Kobayashi, S. (2001). Electrophysiological correlates for response inhibition in a Go/NoGo task. Clinical Neurophysiology, 112(12), 2224-2232. doi:10.1016/S13882457(01)00691-5

Bruin, K. J., \& Wijers, A. A. (2002). Inhibition, response mode, and stimulus probability: A comparative event-related potential study. Clinical Neurophysiology, 113(7), 1172-1182. doi:10.1016/S1388-2457(02)00141-4

Bryden, P. J., Bruyn, J., \& Fletcher, P. (2005). Handedness and health: An examination of the association between different handedness classifications and health disorders. Laterality, 10(5), 429-440. doi:10.1080/13576500442000193

Donkers, F. C. L., \& van Boxtel, G. J. M. (2004). The N2 in go/no-go tasks reflects conflict monitoring not response inhibition. Brain and Cognition, 56(2), 165-176. doi:10.1016/j.bandc.2004.04.005

Enriquez-Geppert, S., Konrad, C., Pantev, C., \& Huster, R.J. (2010). Conflict and inhibition differentially affect the N200/P300 complex in a combined Go/Nogo and stop-signal task. Neurolmage, 51(2), 877-887, doi:10.1016/j.neuroimage.2010.02.043

Falkenstein, M., Koshlykova, N. A., Kiroj, V. N., Hoormann, J., \& Hohnsbein, J. (1995). Late ERP components in visual and auditory Go/Nogo tasks. Electroencephalography and Clinical Neurophysiology, 96(1), 36-43. doi:10.1016/0013-4694(94)00182-K 
Garavan, H., Ross, T. J., \& Stein, E. A. (1999). Right hemispheric dominance of inhibitory control: An event-related functional MRI study. Proceedings of the National Academy of Sciences, 96(14), 8301-8306.

Halpern, D. F., Haviland, M. G., \& Killian, C. D. (1998). Handedness and sex differences in intelligence: Evidence from the medical college admission test. Brain and Cognition, 38(1), 87-101. doi:10.1006/brcg.1998.1021

Heil, M., Osman, A., Wiegelmann, J., Rolke, B., \& Henninghausen, E. (2000). N200 in the Eriksentask: Inhibitory executive process? Journal of Psychophysiology, 14(4), 218-225. doi:10.1027//0269-8803.14.4.218

Ilyina, M. N., \& Ilyin, E. P. (1975). Ob odnom iz uslovii diagnostirovaniya sily nervnoi sistemy po vozbuzhdeniyu s pomoshch'yu tepping-testa [On the one condition of diagnostics of the power of nervous system with the arousal in tapping test]. In Psikhofiziologicheskie osobennosti sportirmoi deyatel'nosti: Sbomik nauchnykh rabot [Psychophysiological characteristics of sport activity: Collected works] (pp. 183-186). Leningrad: LGPI.

Jorgenson, C., Davis, J., Opella, J., \& Angerstein, G. (1980). Hemispheric asymmetry in the processing of Stroop stimuli: an examination of gender, hand-preference, and language differences. Intemational Joumal of Neuroscience, 11(3), 165-169. doi:10.3109/00207458009147582

Khomskaya, E. D., Privalova, N. N., Enikolopova, E. V., Efimova, I. V., Stepanova, O. B., \& Gorina, I. S. (1995). Metody otsenki mezhpolusharnoi asimmetrii $i$ mezhpolusharnogo vzaimodeistviya [Methods of assessment of hemispheric asymmetry and hemispheric intercommunication]. Moscow: Moscow University Press.

Kok, A. (1999). Varieties of inhibition: Manifestations in cognition, event-related potentials and aging. Acta Psychologica, 101(2-3), 129-158. doi:10.1016/S0001-6918(99)00003-7

Lazarev, I. E., \& Kirenskaya, A. V. (2008). The influence of eye dominance on saccade characteristics and slow presaccadic potentials. Human Physiology, 34(2), 150-160.

Lewin, J., Kohen, D., \& Mathew, G. (1993). Handedness in mental handicap: Investigation into populations of Down's syndrome, epilepsy and autism. British Joumal of Psychiatry, 163(5), 674-676. doi:10.1192/bjp.163.5.674

Li, L., Gratton, C., Yao. D., \& Knight, R. T. (2010). Role of frontal and parietal cortices in the control of bottom-up and top-down attention in humans. Brain Research, 1344, 173-184. doi:10.1016/j.brainres.2010.05.016

Logan, G. D. (1994). On the ability to inhibit thought and action: A user's guide to stop signal paradigm. In D. Dagenbach, \& T. H. Carr (Eds.), Inhibitory processes in attention, memory, and language (pp. 189-239). San Diego, CA: Academic Press.

Miyake, A., Friedman, N. P., Emerson, M. J., Witzki, A. H., \& Howerter, A. (2000). The unity and diversity of executive functions and their contributions to complex "frontal lobe" tasks: A latent variable analysis. Cognitive Psychology, 41, 49-100. doi:10.1006/cogp.1999.0734

Perelle, I. B., \& Ehrman, L. (1994). An international study of human handedness: The data. Behavior Genetics, 24(3), 217-227. doi:10.1007/BF01067189

Serrien, D. J., \& Sovijärvi-Spapé, M. M. (2013). Cognitive control of response inhibition and switching: hemispheric lateralization and hand preference. Brain and Cognition, 82(3), 283-290. doi:10.1016/j.bandc.2013.04.013

Simmonds, D. J., Pekar, J. J., \& Mostofsky, S. H. (2008). Meta-analysis of Go/No-go tasks demonstrating that fMRI activation associated with response inhibition is taskdependent. Neuropsychologia, $46,224-232$. 
Julia A. Marakshina - graduate student, Faculty of Psychology, Lomonosov Moscow State University; research fellow, Psychological Institute of Russian Academy of Education.

Research area: psychophysiology, mechanisms of cognitive control, attention and working memory.

E-mail: retalika@yandex.ru

Alexander V. Vartanov - senior research fellow, Faculty of Psychology, Lomonosov Moscow State University, Ph.D.

Research area: psychophysiology, mechanisms semantics of consciousness and higher mental functions.

E-mail: a_v_vartanov@mail.ru

Boris I. Bespalov - senior research fellow, Faculty of Psychology, Lomonosov Moscow State University, Ph.D.

Research area: cultural-activity psychology, psychophysiological problem, logical-mathematical models of cognitive activities.

E-mail: bespalovb@mail.ru 